\title{
Miriam SOLOMON
}

\section{Guest Editor's Introduction}

EPISTEME held its second annual conference at the University of Massachusetts, Amherst, on June 24th and 25th, 2005. In a conference room with a panoramic view of the campus, the Connecticut River Valley and the Berkshire Mountains, some forty people listened to and vigorously discussed seven papers over two days. We dined splendidly at the 1728 Homestead House and continued our discussions at the home of Hilary Kornblith. On behalf of the participants, I thank Hilary for all the local arrangements and the University of Massachusetts and Amherst College for their sponsorship of the conference.

Alvin Goldman approached me in summer 2004 with the idea of organizing a themed conference on "rationality" for EPISTEME's second annual conference on social epistemology. I aimed for a balanced program of invited and contributed papers. We received twenty-one submissions, of which we could accept only three papers (those of Michael Bishop, Simon Evnine and Todd Stewart). I would like to thank the referees: Jonathan Adler, Don Fallis, Richard Feldman, Harold Kincaid, Hilary Kornblith, Christian List and Leslie Marsh for their diligent work in reading and assessing the contributed papers. I wish we could have accepted more of the submissions, which demonstrated the vitality of social epistemology as a field.

As it turned out, the invited and submitted papers jointly cover a range of issues. If we employ the widely mentioned distinction between "conservative social epistemology" and more radical social epistemology, where "conservative social epistemology" refers to investigations of how social practices can benefit the reasoning of individuals, and more radical social epistemology refers to other kinds of evaluation of social practices, then two of the papers lthose by Richard Foley and Todd Stewart) fell into the "conservative social epistemology" category and the others, in their various ways, count as more radical social epistemology. Three of the more radical papers tackled the topic of judgment aggregation, exploring the methods and applications of aggregating group decisions from individual decisions.

Richard Foley opened the conference with his keynote address, "Universal Intellectual Trust." Building on his recent work on self-trust (Foley 2001 1, he argues that intellectual trust of others is, generally speaking, rational because it is a reasonable extension of self-trust. The reasonableness is based on an assessment that people are more similar than they are different; so that, in general, whatever reasons one has for trusting oneself apply towards trusting others.

Todd Stewart addresses the epistemic question raised by "competing social practices." The question is this: if one becomes aware that someone else (perhaps, a member of another culture or groupl makes use of a different epistemic practice in order to reach their beliefs, is the reasonable reaction skepticism about one's own practices? Alston has argued that it is not, unless an external means of assessing the reliability of the competing practice is available, and such assessment is positive. Stewart finds this argument ad hoc, and proposes instead that skepticism is appropriate, provided that the different epistemic practice is real, rather than an imaginary philosophical thought experiment about possible practices.

The first two papers on judgment aggregation recognize that there are different ways of aggregating the judgment of individuals when making group decisions. The outcomes of different methods of aggregation can differ, and no one of these ways is obviously "best." A simple example of this is the difference between the results of the popular vote and the electoral vote in the 2000 U.S. Presidential elections: the popular vote was 
for Al Gore, and the electoral vote for George W. Bush. Philip Pettit and Christian List's recent result, which they call "the impossibility theorem" (List and Pettit 2002) argues that there is no aggregation procedure yielding consistent collective judgments that satisfies four reasonable constraints luniversal domain, completeness, anonymity, systematicityl. This leaves the social choice theorist with several options - to either violate consistency or to violate one or more of the four constraints. Christian List's paper, "Group Knowledge and Group Rationality: A Judgment Aggregation Perspective," explores the different consequences of violating each of the constraints, yielding multiple kinds of aggregation procedure. Don Fallis's paper, "Epistemic Value Theory and Judgment Aggregation" compares the majority vote procedure (which violates consistency) with a consistency maintaining procedure. Fallis argues that neither procedure is "best" if we consider the full range of epistemic values and the full range of cases. In particular, "consistency" does not trump all other epistemic values.

The third paper on judgment aggregation uses a procedure of judgment aggregation for the case of individual persons, whose changing decisions over time may be modeled as individual votes which need to be aggregated in some reasonable way. Simon Evnine reflects that, in general, one's later self is more of an expertmakes better decisions - than one's earlier self (this generality has salient exceptions, such as for memoryl. Thus deference to later selves in aggregating decisions is appropriate and Evnine recommends complete deference to the expert later self (weighting earlier judgments as $\mathrm{O}$ and later judgments as 1). Evnine models each individual person as a group of experts satisfying this "principle of reflection". Reflection is a constitutive norm of persons, so that to the degree that an individual human being fails to satisfy reflection, their personhood is compromised.

Michael Bishop's paper, "The Autonomy of Social Epistemology," argues that, in many situations, we can do better than consult an expert. In fact, individual expert judgments are outperformed by statistical prediction rules (SPRs), even when those rules are based on the criteria for judgment that the experts themselves use, and even when experts have SPRs at their disposal.
The reason for this is that individual experts are often skewed in particular cases by such factors as especially salient evidence and recent experiences /which tend to be weighed more heavily than past experiences), and this leads them to error and, if they are following SPRs, to defect from the SPR on occasion. Bishop's conclusion is that individual reasoning, even when that reasoning is expert reasoning, ought to be superseded by the (group or institutional) application of statistical prediction rules, where available. Statistical prediction rules are socially "autonomous" in that they cannot reasonably be recommendations for individual experts. Individuals, even expert individuals, with the best epistemic will in the world, will still defect from statistical prediction rules in ways that will lead them, overall, to perform worse than statistical prediction rules.

Helen Longino's paper, "Circles of Reason," distinguished three kinds of mental activity: "not reasoning", "reasoning", and "reasoning well". In making these distinctions, she aims to avoid missteps made by moderns and post-moderns. Moderns, to generalize broadly, only say what it is to reason well-typically, to follow "the scientific method" as they describe it. Those who do not reason well are not reasoning at all. So moderns drop out the middle category. This has led, historically, to intolerance of reasonable alternative practices. Post-moderns, also generalizing broadly, view all belief generating mechanisms as on a par, so they collapse all the categories together, and lose the ability to make normative judgments at all. Longino's suggestion is to characterize the middle category, "reasoning," in normative terms, as the ability to distinguish relevant evidence. It is an achievement to be reasoning at all, rather than engaging in some other mental activity. Different epistemic practices may consider evidence in different ways (they may weight some kinds of evidence more heavily than others, for example), but all are reasoning practices so long as they distinguish relevant evidence in their projects of inquiry. "Circles of Reason" may be viewed as another route to Longino's critical contextual empiricism (Longino 2002), which requires of scientific communities that they, at the very least, share the valuing of empirical success. 
Authors were given the opportunity to revise their papers after the conference to address questions and criticisms that were raised in discussion. As guest editor, my role has been to encourage, rather than to require any particular changes. I hope that you enjoy this issue of EPISTEME.

\section{Rererences}

Alston, William (1991). Perceiving God: The Epistemology of Religious Experience. Ithaca: Cornell University Press. Ithaca, NY

Foley, Richard (2001). Intellectual Trust in Oneself and Others. Cambridge: Cambridge University Press.

List, Christian \& Pettit, Philip (2002). Aggregating Sets of Judgments: An Impossibility Result. Economics and Philosophy 18, pp. 89-110.

Longino, Helen (2002). The Fate of Knowledge. Princeton: Princeton University Press.

Miriam Solomon is Professor of Philosophy at Temple University. Her research interests are in philosophy of science, philosophy of medicine, epistemology and feminist philosophy. She is the author of Social Empiricism (2001) and numerous articles in journals and anthologies. 